\title{
Neuroprotection in Experimental Stroke with Targeted Neurotrophins
}

\author{
Dafang Wu \\ Department of Radiology, Wayne State University School of Medicine, Children's Hospital of Michigan, PET Center, \\ Detroit, Michigan 48201
}

\begin{abstract}
Summary: More than 30 neurotrophins have been identified, and many of them have neuroprotective effects in brain ischemia or injury. However, all the clinical trials with several neurotrophins for the treatment of acute ischemic stroke or neurodegenerative diseases have failed so far, primarily because of their poor blood-brain barrier (BBB) permeability. This article is an overview of recent progress in the research focused on BBB targeted neurotrophins using a chimeric peptide approach, in which antitransferrin receptor antibody was used as a BBB delivery vector, and neurotrophin peptide was conjugated to the antibody via the avidin/biotin technology. Vasoactive intestinal peptide was the first model chimeric peptide to show an enhanced CNS effect after noninvasive peripheral administration. Brain-derived neurotrophic factor (BDNF) chimeric peptide was neuroprotective in rats subjected to tran-
\end{abstract}

sient forebrain ischemia, permanent focal ischemia, or transient focal ischemia. Delayed treatments with the BDNF chimeric peptide showed an effective time window of 1-2 h after ischemia. Basic FGF chimeric peptide was highly effective in the reduction of infarct volume in the rat model of permanent focal ischemia, with lowest effective dose of $1 \mu \mathrm{g}$ per rat. Future studies in this exciting area include genetically engineered fusion proteins or humanized antibodies for BBB drug targeting with less immunogenicity and reduced working burden in the chemical conjugation, the use of antihuman insulin receptor antibody for higher BBB delivery efficiency, and combination therapies using chimeric neurotrophins plus other neuroprotectants to achieve additive or synergistic effects. Key Words: Blood-brain barrier, neurotrophins, drug targeting, transferrin receptor, ischemic stroke, neuroprotection.

\section{ISCHEMIC STROKE AND THROMBOLYTIC THERAPIES}

Stroke is the third leading cause of death in the developed countries and the leading cause of major adult disability. It was projected that, if the age-specific rates of stroke remain unchanged, the overall number of strokes in the United States will increase from approximately 700,000 in 2002 to 1136,000 in 2025 , owing to the rising percentage of the population in older groups. ${ }^{1}$ Among the stroke patients, $85-90 \%$ of the cases are ischemic stroke with a predominant cause of cerebral arterial thrombosis. ${ }^{2}$ Over the last decade, significant progresses have been made in the development of thrombolytic therapies for acute ischemic stroke. The first was the Food and Drug Administration-approved tissue plasminogen activator (tPA) in 1996. The benefits of tPA

Address correspondence and reprint requests to Dr. Dafang Wu, M.D., Ph.D., Children's Hospital of Michigan, PET Center, Room GP-112,3901 Beaubien Boulevard, Detroit, MI48201.E-mail: dwu@ pet. wayne.edu. therapy were demonstrated in a National Institute of Neurological Disorders and Stroke-sponsored clinical trial. ${ }^{3}$ The study showed that intravenous administration of tPA within $3 \mathrm{~h}$ of the onset of acute ischemic stroke improved clinical outcome at 3 months. Another advance was the demonstration that intra-arterial administration of prourokinase (proUK) initiated within $6 \mathrm{~h}$ of stroke onset was also beneficial for patients with middle cerebral arterial occlusion. ${ }^{4}$ So far, the proUK therapy remains to be approved, and the need for skilled neuroradiological expertise is likely to limit its application in major medical centers as a second-line rescue therapy for patients who have failed intravenous tPA thrombolysis. ${ }^{5}$ With the restrictive time window of $3 \mathrm{~h}$, only $1-2 \%$ of patients with acute ischemic stroke are able to receive the tPA therapy. ${ }^{6}$ In addition, thrombolytic therapies have increased risks of cerebral hemorrhage, which limits their applications for certain patients. Clearly, there is a crucial need for the development of neuroprotective therapies, which could be implemented alone, or in combination with thrombolytic approaches to improve clinical outcome of more patients with acute ischemic stroke. 


\section{CLINICAL TRIALS OF NEUROTROPHINS}

The strategy of neuroprotection against ischemic brain injury has been always prioritized in the research of new therapies for acute stroke. However, so far, all neuroprotective trials have failed to show any benefits in humans. The basic research and clinical trials of neuroprotective agents have been intensively reviewed recently. ${ }^{7-9} \mathrm{Al}-$ though there are multiple underlying reasons for the failures, the blood-brain barrier (BBB) is an outstanding issue and will continue to be a hurdle in the future research and development of new therapies for acute ischemic stroke. The BBB is a natural barrier that keeps large molecules from entering the brain. Although cerebral ischemia causes disruption of the $\mathrm{BBB}$, it occurs only after several hours of the onset. ${ }^{10}$ Because the therapeutic time window for most neuroprotective agents is less than $6 \mathrm{~h},{ }^{11}$ all the large molecule therapeutics cannot enter the brain from blood stream to achieve a pharmacological level within the brain, and this is especially true for neurotrophins, which are endogenous large molecule peptides or proteins. In the case of neurodegenerative diseases, such as Alzheimer's and amytrophic lateral sclerosis (ALS), the BBB appears intact, and keeps neurotrophins, as described below, from reaching targeted neurons to exert therapeutic effects after peripheral administration. $^{12}$

Nerve growth factor (NGF), as the name implies, is an endogenous neurotrophic factor for central cholinergic neurons, which are depleted in Alzheimer's disease. ${ }^{13,14}$ In animal studies mainly involving rodents, intracerebroventricular administration of NGF can rescue the cholinergic neurons, stimulate axonal growth, and improve cholinergic function. ${ }^{15-17}$ Similarly, intracerebroventricular injection of NGF attenuated neuronal death in the hippocampi of gerbils subjected to brain ischemia. ${ }^{18}$ Encouraged by these preclinical results, a female Alzheimer patient received NGF treatment in $1991 .{ }^{19}$ This represented the first case of clinical trial with neurotrophin. Knowing the poor BBB penetration of NGF after peripheral administration, the patient received NGF via a slow intracerebroventricular infusion for a period of 3 months! Even so, the patient failed to show any sustained improvement in memory or cognitive functions. The reason for the failure is because of the bulk-flow of the cerebral spinal fluid (CSF) and the extremely slow rate $\left(10^{-6}\right.$ $\mathrm{cm} / \mathrm{sec}$ ) of solute diffusion from CSF into brain tissue that severely limits the penetration of intracerebroventricular administrated NFG into the brain parenchyma where the cholinergic neurons were supposed to be targeted. ${ }^{20}$

BDNF is a neurotrophin that promotes the survival and growth of developing neurons in vitro ${ }^{21,22}$ and improves motor neuronal functions in animal models. ${ }^{23,24}$ In animals subjected to transient forebrain ischemia, BDNF attenuated ischemic neuronal injury. ${ }^{25}$ These ex- perimental data prompted multiple phases of clinical trials with BDNF for the treatment of more than 1000 patients with ALS, a motor neuron degenerative disease. BDNF was administrated either via subcutaneous injection $^{26,27}$ or through intrathecal infusion. ${ }^{28}$ None of the trials showed any beneficial effects for ALS patients. The reason for the failure of the study using intrathecal administration is the same as the intracerebroventricular infusion of NGF, which was discussed earlier. The trials of BDNF after subcutaneous injection are unlikely to succeed, because of the poor penetration of BDNF across the BBB and because of unfavorable pharmacokinetics of the peptide (plasma half-life $<10 \mathrm{~min}$ ). ${ }^{29}$

Basic FGF (bFGF or FGF2) is probably the most potent neurotrophic factor in the FGF superfamily. ${ }^{30}$ bFGF is widely distributed in neuronal and non-neuronal tissues and has additional effects of mitogenic activity and vasodilatation. ${ }^{31,32}$ There is a growing body of evidence that bFGF is neuroprotective in a variety of preclinical studies, and data in this area had been reviewed by Ay and associates. ${ }^{33}$ It should be noted that bFGF is a cationic peptide that undergoes transport across the BBB at a modest rate via absorptive-mediated transcytosis after intravenous administration. ${ }^{34}$ This may explain why peripheral administration of bFGF at high doses $(135-200 \mu \mathrm{g} / \mathrm{kg}$, i.v. infusion over $3 \mathrm{~h}$ ) produced moderate neuroprotection (24-50\% reduction of infarct volume in animals subjected to middle cerebral arterial occlusion). ${ }^{33}$ However, a phase III clinical trial of bFGF in human subjects with acute ischemic stroke was stopped, owing to higher mortality rates in the treated groups as compared with the control group. ${ }^{9,35}$ A recent European-Australian phase II/III trial with bFGF (5-10 $\mathrm{mg}$, i.v. infusion over $24 \mathrm{~h}$ ) in a total of 286 patients with acute ischemic stroke confirmed that intravenous administration of bFGF did not produce any significant neuroprotection, instead caused dose-dependent hypotension and had an increased mortality rate in the treated patients. ${ }^{36}$ The failure of these clinical trials with bFGF exemplifies the importance of BBB drug targeting to achieve a pharmacologically significant level of therapeutics in the brain while minimizing side effects in the peripheral. This strategy will be discussed in details below.

\section{CHIMERIC PEPTIDE APPROACHES FOR TARGETING TRANSFERRIN RECEPTORS ON THE BBB}

Brain drug delivery strategies can be generally categorized as 1) neurosurgical, 2) pharmacologic, and 3) physiologic approaches. ${ }^{20}$ The most commonly used neurosurgical approach is the intracerebroventricular injection or perfusion. As discussed earlier, this method is invasive, and the intracerebroventricular route may be 
effective for the treatment of diseases of brain surface, such as meningitis, but not useful for targeting to the brain parenchyma because of the rapid bulk-flow of CSF, and extremely slow diffusion rate of solutes from the CSF into brain parenchyma. Pharmacologic approaches include liposomes and peptide lipidization. Because the BBB has a size exclusion (molecular weight, 300-500), these approaches are unlikely to be effective for the transport of neurotrophins (molecular weight usually over 10,000). By contrast, physiologic approaches for BBB delivery of peptide or protein drugs use a chimericpeptide strategy, wherein a nontransportable peptide drug, such as a neurotrophin, is conjugated to another peptide or protein that functions as a brain drug delivery carrier or vector. The model vector is the murine monoclonal antibody $(\mathrm{mAb})$ against rat transferrin receptors designated as OX26. The transferrin receptors are highly expressed on the brain capillary endothelial cells, ${ }^{37}$ which make up of the BBB in vivo. The binding of the OX26-neurotrophin conjugate to an epitope on the transferrin receptor triggers physiologic internalization, and receptor-mediated transcytosis through the BBB. The conjugation of the OX26 mAb to a neurotrophin is facilitated by the use of avidin or streptavadin technology. ${ }^{20}$ This chimeric peptide approach has been successfully used for BBB delivery of several neurotrophins or peptide drugs, for which enhanced CNS pharmacologic effects or neuroprotection have been consistently demonstrated in a variety of animal models including brain ischemia. ${ }^{38-43}$

\section{TARGETED VASOACTIVE INTESTINAL PEPTIDE FOR ENHANCEMENT OF CNS EFFECTS}

Vasoactive intestinal peptide (VIP) is a 28-amino acid polypeptide that is widely distributed in gastrointestinal tissues, and the central and peripheral nervous system. ${ }^{44}$ VIP has a variety of biological activities and plays a significant role in the regulation of cerebral blood flow. ${ }^{45,46}$ In addition, VIP has been demonstrated to have potent neuroprotective effects against human immunodeficiency virus gp120 protein, tetrodotoxin, amyloid $\beta$ peptides, and NMDA (an excitotoxin). ${ }^{47-49}$ VIP can cause cerebral vasodilatation when directly applied to brain arterial preparations. ${ }^{45,46}$ However, no cerebral vasodilatation was observed when VIP was infused into the carotid artery, owing to the failure of VIP to cross the $\mathrm{BBB}$ in vivo. ${ }^{20,50}$

To facilitate VIP transport across the BBB in vivo, a VIP analog was monobiotinylated (abbreviated as bioVIPa), and the OX26 mAb was initially conjugated to avidin. ${ }^{51}$ On the day of experiment, the bio-VIPa was added to the OX26-avidin, and a VIP chimeric peptide, e.g., bio-VIPa/OX26-avidin, was formed immediately

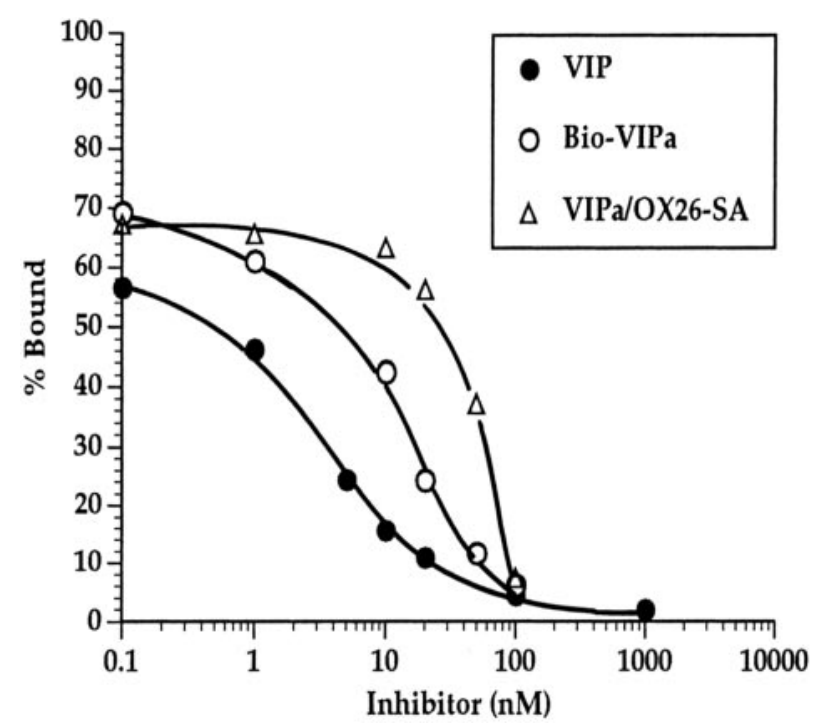

FIG. 1. Competition curves of three VIP analogs in the radioreceptor binding assays using rat lung membrane preparation as the VIP receptor source, and [I-125] VIP as the tracer. Each point on the graph represents the mean of duplicate incubations, which varied less than $10 \%$. Adapted with permission from Wu and Pardridge. Central nervous system pharmacologic effect in conscious rats after intravenous injection of a biotinylated vasoactive intestinal peptide analog coupled to a blood-brain barrier drug delivery system. J Pharmacol Exper Ther 279:77-83. Copyright $\odot$ 1996, American Society for Pharmacology and Experimental Therapeutics. All rights reserved. ${ }^{39}$ bio-VIPa $=$ monobiotinylated VIP analog; VIPa/OX26-SA = complex of bio-VIPa and a conjugate of SA and the OX26 mAb to the rat transferrin receptor.

due to prompt avidin/biotin binding. After intracarotid artery infusion at a dose of $12 \mu \mathrm{g} / \mathrm{kg}$, the VIP chimeric peptide produced a $65 \%$ increase in hemispheric brain blood flow in anesthetized rats, whereas equivalent dose of unconjugated VIP analog did not show any CNS effects. ${ }^{51}$ The intra-arterial infusion of the VIP chimeric peptide was necessitated for pharmacokinetic reasons because the introduction of avidin significantly increased the rate of blood clearance of the VIP chimeric peptide after intravenous injection. As a result, the plasma AUC (area under the curve) of the VIP chimeric peptide was markedly reduced as compared with that of unconjugated OX26 mAb. ${ }^{52}$ This pharmacokinetic problem was eliminated by the use of streptavidin (SA), a neutral form of avidin, which keeps the avidin/biotin binding property, but has a slower blood clearance and an enhanced plasma AUC. ${ }^{53}$

After optimization of the pharmacokinetics of the BBB delivery vector, e.g., OX26-SA, the biological activity of the chimeric peptide, e.g., bio-VIPa/OX26-SA, was evaluated by a radioreceptor binding assay. ${ }^{39}$ As shown in Figure 1, the VIP receptor binding affinity was retained in the nanomolar range after biotinylation and subsequently bound to the vector OX26-SA. Subsequent studies showed the brain uptake of the chimeric peptide, bio-VIPa/OX26-SA, was significantly enhanced after in- 

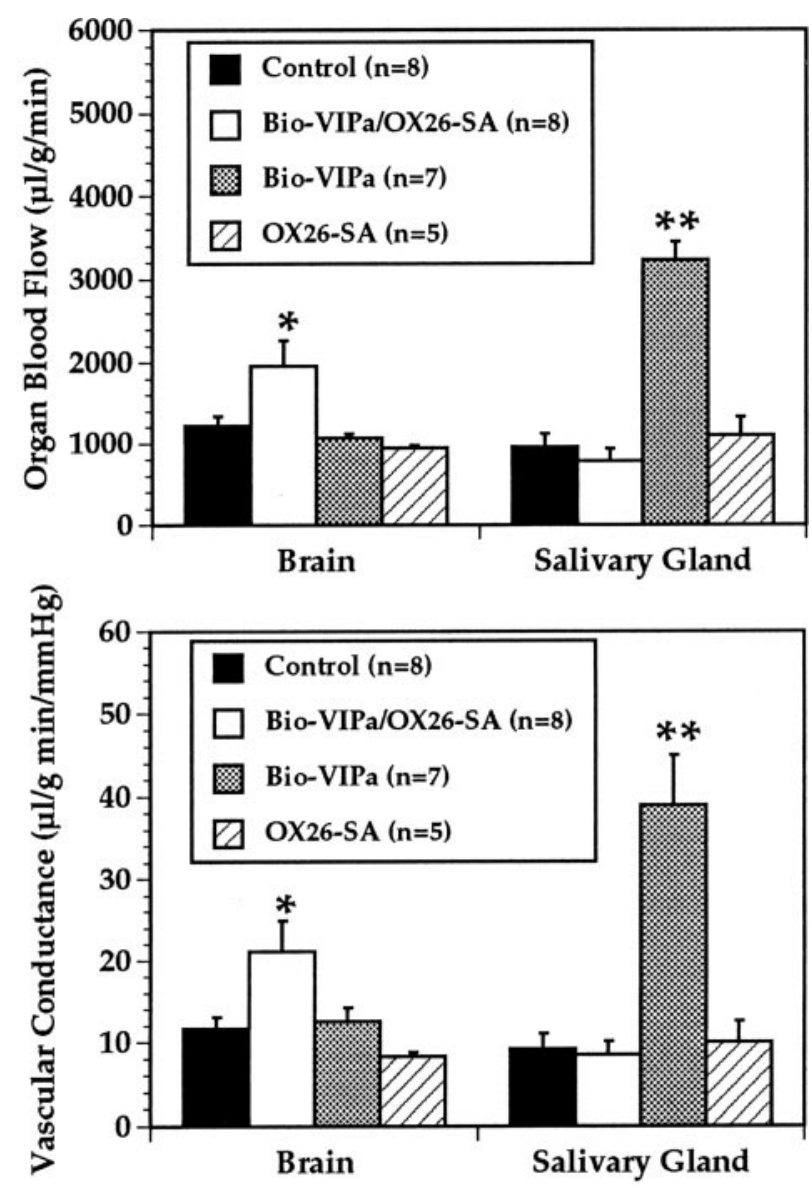

FIG. 2. Effects of the bio-VIPa and chimeric peptide on organ blood flow (top panel), and vascular conductance (bottom panel) in conscious rats. Data are expressed as the mean \pm SEM of the group. ${ }^{*}, p<0.05 ;{ }^{* *}, p<0.01$ as compared with the control group by the use of Student's $t$ test. Adapted with permission from Wu and Pardridge. Central nervous system pharmacologic effect in conscious rats after intravenous injection of a biotinylated vasoactive intestinal peptide analog coupled to a bloodbrain barrier drug delivery system. J Pharmacol Exper Ther 279: 77-83. Copyright $\odot$ 1996, American Society for Pharmacology and Experimental Therapeutics. All rights reserved. ${ }^{39} \mathrm{Bio}-\mathrm{VIPa}=$ monobiotinylated VIP analog; bio-VIPa/OX26-SA = complex of bio-VIPa and a conjugate of SA and OX26 mAb to the rat transferrin receptor. OX26-SA = conjugate of the rat transferrin receptor $\mathrm{mAb}$ and SA with no bio-VIPa attached.

travenous administration, owing to the increased plasma AUC and BBB permeability-surface area product (PS). One hour after intravenous injection, the brain uptake expressed as percentage of injected dose (ID) per gram was nearly ten times higher than that of bio-VIPa without conjugated to OX26-SA $(0.119 \%$ vs $0.013 \%$ ID/g). The CNS effects, e.g., vascular conductance and cerebral blood flow, of the VIP chimeric peptide, were tested in conscious rats after intravenous injection in comparison with unconjugated bio-VIPa or OX26/SA as controls. Administration of the unconjugated bio-VIPa increased salivary gland blood flow by $350 \%$, whereas there was no effect on cerebral blood flow and vascular conductance (FIG. 2). In contrast, bio-VIPa/OX26-SA at an equal dose $(20 \mu \mathrm{g} / \mathrm{kg})$ after intravenous injection to conscious rats caused a marked increase in cerebral blood flow by $60 \%$, which was statistically significant $(p<$ 0.05). Moreover, bio-VIPa/OX26-SA had no significant effects on peripheral organ blood flow, such as salivary gland. The lack of peripheral effects of the chimeric peptide is probably due to the large molecular size of the chimeric peptide (200,000 Da vs 3817 Da for VIP) limiting its diffusion into exocrine tissues such as salivary gland. Therefore, conjugation of the peptide drug to the BBB delivery vector using a avidin/biotin technology makes it possible to achieve enhanced brain uptake leading to CNS therapeutic effects, while restricting drug uptake by peripheral tissues, and thereby reducing side effects or toxicity. The overall therapeutic index of the VIP chimeric peptide was increased by an order of magnitude. As discussed before, VIP has potent vasodilatation and neuroprotection, both effects could be beneficial for the treatment of ischemic stroke, provided VIP is able to cross the BBB to achieve a pharmacologic level in the brain after peripheral administration. Although we did not test the neuroprotection of VIP in an experimental ischemic model, the data presented here clearly showed enhanced brain uptake and improved therapeutic index after conjugation of VIP to the BBB peptide drug delivery vector, e.g., OX26-SA.

\section{TARGETING BDNF FOR TREATMENT OF ANIMALS SUBJECTED TO GLOBAL AND REGIONAL BRAIN ISCHEMIA}

BDNF is an endogenous peptide with very short plasma half-life $(<10 \mathrm{~min}){ }^{29}$ Therefore, there are two major limiting factors that need to be overcome to achieve neuroprotection in vivo after peripheral administration of BDNF. One is the poor BBB permeability, and another is the unfavorable pharmacokinetics. The BDNF was modified by attachment of 2000-Da strands of polyethylene glycol $\left(\mathrm{PEG}^{2000}\right)$ to surface carboxyl groups. ${ }^{54}$ This modification is termed pegylation and results in prolonged plasma circulation of the neurotrophic factor. The pegylated BDNF was conjugated to the OX26 mAb with the use of avidin/biotin technology as described before. In this approach, a single biotin moiety was attached to the tip of one of the PEG strands conjugated to the BDNF. The BBB delivery vector OX26-SA can immediately capture the biotinylated BDNF-PEG ${ }^{2000}$. The combined conjugate, designated BDNF-PEG ${ }^{2000}$-biotin/OX26-SA, has an improved plasma pharmacokinetics and BBB PS, relative to unconjugated BDNF. ${ }^{54}$ After the multiple modifications, the biological activity of the BDNF chimeric peptide was evaluated using a cell survival study and the TrkB autophosphorylation assay. Both BDNF-PEG ${ }^{2000}$-biotin and 
A

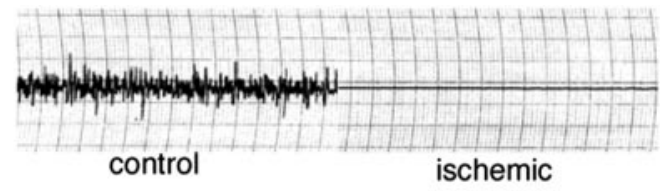

B

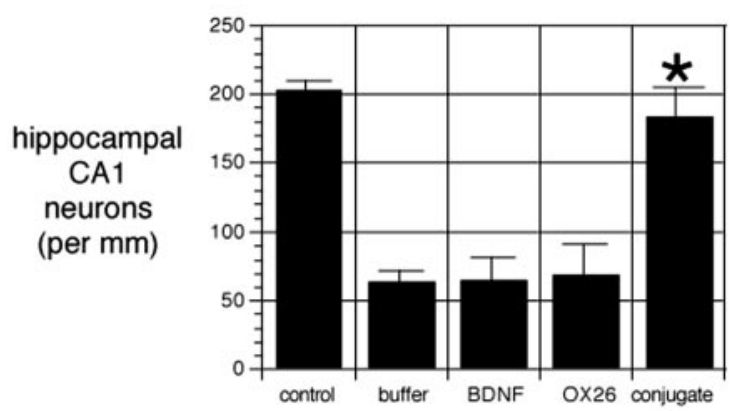

FIG. 3. A: EEG for rats before ischemia (control section) and isoelectric EEG during the 12-min ischemic period. B: Hippocampal CA1 neuron density in control animals not subjected to transient forebrain ischemia and in four groups of animals with 12-min transient forebrain ischemia confirmed by the isoelectric EEG. These four groups of animals were treated intravenously with either buffer, unconjugated BDNF, OX26 without BDNF attached, or BDNF-PEG ${ }^{2000}$-biotin conjugated to OX26/SA, respectively. Only the latter group resulted in a statistically significant $\left({ }^{*}, p<0.0025\right)$ therapeutic effect after intravenous administration. Adapted with permission from $\mathrm{Wu}$ and Pardridge. Neuroprotection with noninvasive neurotrophin delivery to the brain. Proc Natl Acad Sci USA 96:254-259. Copyright ๑ 1999 , National Academy of Sciences. All rights reserved. ${ }^{40}$
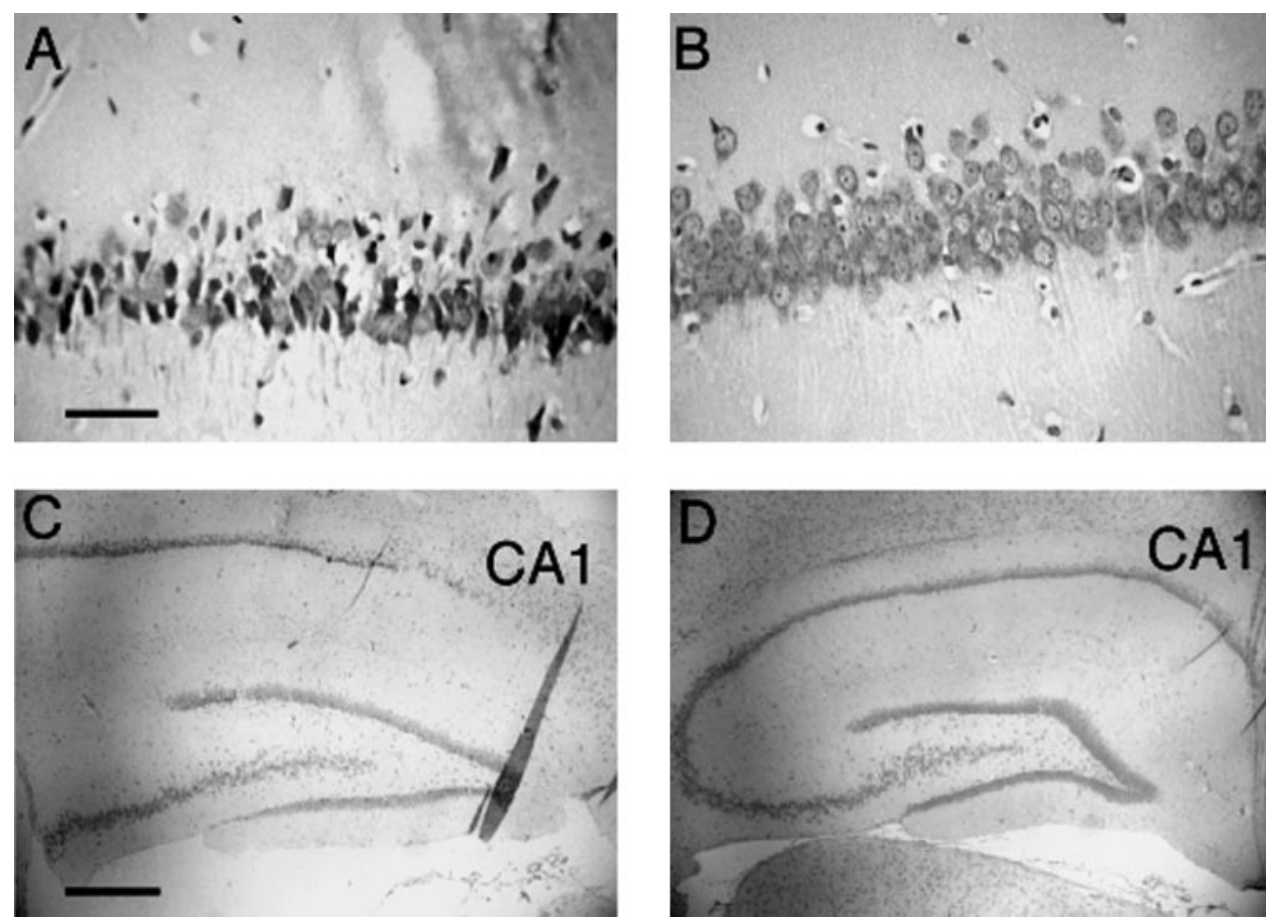

FIG. 4. Nessl staining of hippocampal CA1 sector 7 days after transient forebrain ischemia sufficient to induce isoelectric EEG in the rat. Intravenous treatment immediately after the ischemia and daily for 7 days consisted of unconjugated BDNF (A and C), or BDNF-PEG 2000-biotin conjugated to OX26/SA (B and D). The CA1 neuronal density is markedly reduced in the animals treated with unconjugated BDNF but is normalized by the treatment with the BDNF-PEG ${ }^{2000}$-biotin/OX26-SA conjugate. Magnification bars: 21 (A) and 212 (C) $\mu \mathrm{m}$. Adapted with permission from Wu and Pardridge. Neuroprotection with noninvasive neurotrophin delivery to the brain. Proc Natl Acad Sci USA 96:254-259. Copyright $\odot$ 1999, National Academy of Sciences. All rights reserved. ${ }^{40}$ 
focal brain ischemia. ${ }^{42,43}$ In the first project, the rats were subjected to permanent middle cerebral artery occlusion (MCAO), and treatment with the BDNF chimeric peptide was initiated immediately via intravenous injection. The BDNF chimeric peptide produced a dose-dependent neuroprotection as evidenced by infarct volume reduction up to $65 \%$, whereas equal doses of unconjugated BDNF and the OX26 mAb were ineffective. Delayed treatments showed that the BDNF-mediated neuroprotection in the permanent MCAO model has an effective window of 1-2 $\mathrm{h}$ after the ischemia, although the pharmacologic effect was progressively diminished in proportion to the time delay between MCAO and treatment. ${ }^{38,42}$ The second project was designed to test whether the BDNF chimeric peptide was still effective after delayed intravenous administration ( $1 \mathrm{~h}$ after ischemia) in an animal model for transient focal brain ischemia, e.g., $1 \mathrm{~h}$ of MCAO followed by reperfusion. Although the treatment was given by a single dose, the neuroprotective effects were observed at either $24 \mathrm{~h}$ or 7 days later, with infarct volume reduction of $68 \%$, and $70 \%$, respectively. ${ }^{43}$ The results indicated that the neuroprotective effects of the BDNF chimeric peptide in the animal model of transient MCAO was long lasting and persisted for at least 7 days. One interesting finding was that the BDNF chimeric peptide was only effective in the reduction of cortical infarct, but not subcortical injury. This is consistent with prior finding by Schabitz and associates, ${ }^{55}$ indicating that the neuroprotection of BDNF is largely cortical.

\section{BBB TARGETING OF BFGF FOR NEUROPROTECTION AGAINST REGIONAL BRAIN ISCHEMIA}

Human bFGF is a potent neurotrophic factor that has been consistently demonstrated to be neuroprotective in a variety of in vitro tests and in vivo studies using different animal models of brain ischemia. ${ }^{33}$ However, the dose of intravenous bFGF that was required to achieve neuroprotection in the brain was relatively high, and the peripheral side effects, which are apparently dose dependent, have stopped clinical trials of bFGF as a neuroprotective agent. ${ }^{35,36}$ The high peripheral doses are required because the transport of bFGF (molecular weight of $16,400)$ across the BBB is slow and limited to absorptive-mediated transcytosis of this cationic peptide. ${ }^{34}$

Human recombinant bFGF was monobiotinylated and the product was termed as bio-bFGF. In parallel, the OX26 mAb was conjugated to SA via a stable thio-ether bond. On the day of experiment, bio-bFGF was added to the conjugate OX26-SA and was immediately captured by the SA due to the binding property of avidin/biotin. The bFGF chimeric peptide showed a retained receptor binding affinity and had an improved plasma pharmacokinetics and BBB permeability. ${ }^{41}$ Then, the neuroprotec-

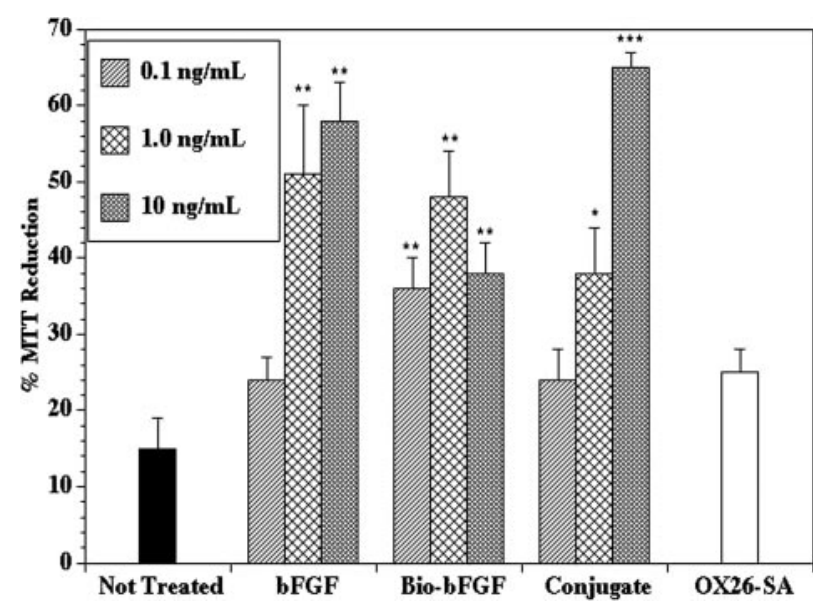

FIG. 5. Neuroprotective effect of bFGF analogs in the mixed rat forebrain cortical cell cultures subjected to hypoxia $(24 \mathrm{~h}) /$ reoxygenation (4 h) in the MTT (3-(4,5-dimethylthiazol-2-yl)-2,5-diphenyltetrazolium bromide) assay. Each bar in the graph represents mean \pm SD of triplicate incubations. * $p<0.05 ;{ }^{* \star}, p<0.01$; ${ }^{* \star *}$, $p<0.001$ as assessed with Student's $t$ test. Adapted with permission from Song et al. Enhanced neuroprotective effects of basic fibroblast growth factor in regional brain ischemia after conjugation to a blood-brain barrier delivery vector. $J$ Pharmacol Exper Ther 301:605-610. Copyright $\odot$ 2002, American Society for Pharmacology and Experimental Therapeutics. All rights reserved. ${ }^{56}$ bio-bFGF = biotinylated bFGF; Conjugate = complex of bio-bFGF and a conjugate of the OX26 mAb to the rat transferrin receptor and SA; OX26-SA = no bFGF attached.

tive effects of bFGF chimeric peptide against hypoxia/ reoxygenation injury were evaluated using the mixed rat forebrain cortical cell cultures. ${ }^{56}$ As shown in Figure 5, both bio-bFGF and bio-bFGF/OX26-SA exhibited comparable neuroprotection in a dose-dependent manner as did the unconjugated bFGF. By contrast, OX26-SA did not show any significant neuroprotection in the same cultures. Subsequently, the bFGF chimeric peptide was intravenous administrated to rats subjected to permanent MCAO, whereas equal doses of bFGF, or the OX26 $\mathrm{mAb}$ were also given to different animals as controls. A single intravenous injection of the bFGF chimeric peptide, equivalent to a dose of $25 \mu \mathrm{g} / \mathrm{kg} \mathrm{bFGF}$, produced an $80 \%$ reduction in infarct volume, whereas the equivalent doses of unconjugated bFGF or the OX26 mAb did not exhibit any significant neuroprotection in the same model (see FIG. 6). The neuroprotection was time dependent, and there was a $67 \%$ reduction in infarct volume even if the bFGF chimeric peptide was administrated $60 \mathrm{~min}$ after MCAO, whereas no significant effects were observed if the treatment was delayed beyond $2 \mathrm{~h}$. Moreover, at an equivalent dose of $5 \mu \mathrm{g} / \mathrm{kg}$, e.g., approximately $1 \mu \mathrm{g}$ per rat (body weight of $200 \mathrm{~g}$ ), the bFGF chimeric peptide still exhibited neuroprotection as evidenced by $34 \%$ reduction of infarct volume, which was statistically significant $(p<0.01)$. As compared with the prior studies using the same MCAO model, ${ }^{33}$ this study using BBB targeting strategy achieved a reduced dose $(25 \mu \mathrm{g} / \mathrm{kg}$ of bFGF chimeric peptide $v s 135-200 \mu \mathrm{g} / \mathrm{kg}$ of 


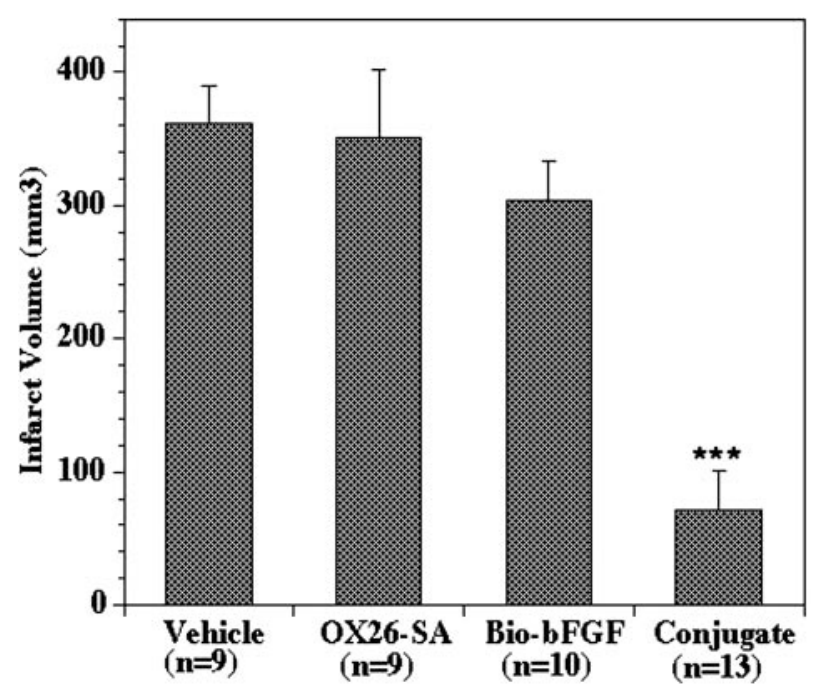

FIG. 6. Reduction of infarct volume in an MCAO model in the rats treated with bFGF chimeric peptide (the conjugate group). Each bar in the graph represents the mean \pm SD of the group. ${ }^{* * *}, p<0.01$ compared with the vehicle group (ANOVA with Bonferroni correction). Adapted with permission from Song et al. Enhanced neuroprotective effects of basic fibroblast growth factor in regional brain ischemia after conjugation to a blood-brain barrier delivery vector. $J$ Pharmacol Exper Ther 301:605-610. Copyright $\odot$ 2002, American Society for Pharmacology and Experimental Therapeutics. All rights reserved. ${ }^{56}$ Bio-bFGF $=$ biotinylated bFGF; Conjugate = complex of bio-bFGF and a conjugate of the OX26 mAb to the rat transferrin receptor and $\mathrm{SA}$; OX26-SA = no bFGF attached.

unconjugated $\mathrm{bFGF}$ ) and an enhanced neuroprotective effect ( $80 \%$ infarct volume reduction versus $24-50 \%$ in the prior studies using unconjugated bFGF). This is probably one of the highest potencies we have ever demonstrated so far in terms of neuroprotective effect in living animals following noninvasive peripheral administration of a neurotrophic factor.

\section{FUTURE PERSPECTIVES}

Although the results in the use of the chimeric peptide approach for the treatment of experimental brain ischemia are very promising, there is still a big gap between these laboratory studies and clinical application of the chimeric neurotrophin peptide for treatment of patients with acute ischemic stroke. One of the concerns is the immunogenicity of the antitransferrin receptor antibody that is used as the BBB peptide drug delivery vector. This problem can be solved or ameliorated by the use of humanized antibody via genetic engineering. ${ }^{57}$ Humanized antibodies are less likely to provoke an immune reaction in human subjects than are murine-derived antibodies. Another related advance is the use of genetically engineered fusion proteins containing avidin and antitransferrin receptor antibody. ${ }^{58}$ The fusion proteins are expected to be bifunctional, e.g., the high binding affinity to the transferrin receptors to trigger transcytosis across the BBB, and the capacity of capturing of any biotinylated large molecular therapeutics to form a chimeric peptide or protein. The success of this project will greatly reduce the working burden of investigators in the tedious preparation of the chimeric peptide with multiple steps of conjugation.

As discussed earlier, the side effects of most of neurotrophins are dose dependent. One strategy to minimize side effects is to reduce the dose as much as possible, and this can be achieved by the chimeric peptide strategy for BBB drug targeting, but the BBB delivery efficacy needs to be improved to further reduce the dose of neurotrophins. So far, most of the studies were carried out using mouse monoclonal antibody against rat transferrin receptor. Recent work, however, has shown that mouse antihuman insulin receptor (HIR), designated as 83-14, has severalfold greater BBB permeability in primates, as compared with the brain uptake of the OX26 mAb in rats. ${ }^{59,60}$ The HIR mAb has been genetically engineered to form a human/mouse chimeric $\mathrm{mAb}$, and the affinity of the chimeric $\mathrm{mAb}$ for the HIR is identical to that of the original murine mAb. ${ }^{61}$ The use of the chimeric HIR $\mathrm{mAb}$ is likely to further reduce the dose of neurotrophin while maintaining neuroprotective effects. Also, the use of the chimeric HIR mAb makes it more feasible for future clinical studies with BBB targeted neurotrophins for acute ischemic stroke because the data derived from the studies in primates are more predictive for effects in humans.

The pathology of ischemic stroke is a complex, and it's unlikely to cure the disease with a single therapeutic agent or approach. There is an increasing interest in the combination therapies for patients with ischemic stroke. ${ }^{62-64}$ Several groups of researchers have already tested the strategy of combined therapies, including therapy using two small molecular neuroprotective agents, ${ }^{62,65}$ dual antiplatelet therapy, ${ }^{66}$ and treatment with combined small molecular neuroprotective agent (citicoline) and neurotrophin, e.g., unconjugated bFGF. ${ }^{67}$ It is anticipated that combination therapies using two chimeric neurotrophin peptides would have additive or synergistic effects in the future treatment of experimental ischemic stroke.

Acknowledgments: The author is grateful to the guidance and inspiration provided by Dr. William Pardridge, M.D. A Scientist Development Grant to the author from the American Heart Association, Western States Affiliate, supported the bFGF work cited in this article. This work was supported in part by National Institutes of Health Grant NS34698.

\section{REFERENCES}

1. Broderick JP. William M. Feinberg Lecture: stroke therapy in the year 2025. Burden, breakthroughs, and barriers to progress. Stroke 35:205-211, 2004. 
2. Fieschi C, Argentino C, Lenzi GL, Sacchetti ML, Toni D, Bozzao L. Clinical and instrumental evaluation of patients with ischemic stroke within the first six hours. J Neurol Sci 91:311-322, 1989.

3. The National Institute of Neurological Disorder and Stroke rt-PA Stroke Study Group. Tissue plasminogen activator for acute ischemic stroke. N Engl J Med 333:1581-1587, 1995.

4. Furlan A, Higashida R, Wechsler L, Gent M, Rowley H, Kase C, et al. Intra-arterial prourokinase for acute ischemic stroke. The PROACT II study: a randomized controlled trial. Prolyse in acute ischemic cerebral thromboembolism. JAMA 282:2003-2011, 1999.

5. Ford GA, Bryant CA, Mangoni AA, Jackson SHD. Stroke, dementia, and drug delivery. Br J Clin Pharmacol 57:15-26, 2004.

6. Hacke W, Brott T, Caplan LR, Meier D, Fieschi C, von Kummer $\mathrm{R}$, et al. Thrombolysis in acute ischemic stroke: controlled trials and clinical experience. Neurology 53[Suppl]:S3-S14, 1999.

7. Hinkle JL, Bowman L. Neuroprotection for ischemic stroke. J Neurosci Nurs 35:114-118, 2003.

8. Ovbiagele B, Kidwell CS, Starkman S, Saver JL. Neuroprotective agents for the treatment of acute ischemic stroke. Curr Neurol Neurosci Rep 3:9-20, 2003.

9. Wahlgren NG, Ahmed N. Neuroprotection in cerebral ischemia: Facts and fancies-the need for new approaches. Cerebrovascular Dis 17[Suppl 1]:153-166, 2004.

10. Menzies SA, Betz AL, Hoff JT. Contributions of ions and albumin to the formation and resolution of ischemic brain edema. $\mathrm{J} \mathrm{Neu}$ rosurg 78:257-266, 1993.

11. Keep RF. Editorial comment. Stroke 32:1381-1382, 2001.

12. Hefti F. Pharmacology of neurotrophic factors. Annu Rev Pharmacol Toxicol 37:239-267, 1997.

13. Hefti F, Hartikka JA, Montero CN, Junard EO. Role of nerve growth factor in the central nervous system. In: Neurobiology of amino acid, peptides, and trophic factors (Ferrendelli JA, Collins RC, Johnson EM, eds), pp 127-138. Norwell, MA: Kluwer Academic Publishers, 1988.

14. Levi-Montalcini R, Angeletti PU. Nerve growth factor. Physiol Rev 48:534-569, 1968.

15. Hefti F. Nerve growth factor promotes survival of septal cholinergic neurons after fimbrial transactions. J Neurosci 6:2155-2162, 1986.

16. Will B, Hefti F. Behavioral and neurochemical effects of chronic intravenous injection of nerve growth factor in adult rats with fimbria lesions. Behav Brain Res 17:17-24, 1985.

17. William L, Varon S, Peterson G, Wictorin K, Bjorklund A, Gage F. Continuous infusion of nerve growth factor prevents basal forebrain neuronal death after fimbria fornix transection. Proc Natl Acad Sci USA 33:9231-9235, 1986.

18. Shigeno T, Mima T, Takakura K, Graham DI, Kato G, et al. Amelioration of delayed neuronal death in the hippocampus by nerve growth factor. J Neurosci 11:2914-2919, 1991.

19. Olson L, Nordberg A, von Holst H, Backman L, Ebendal T, Alafuzoff I, et al. Nerve growth factor affects C-11 nicotine binding, blood flow, EEG, and verbal episodic memory in an Alzheimer patient (case report). J Neural Transm 4:79-95, 1992.

20. Pardridge WM. Peptide drug delivery to the brain. New York: Raven Press, 1-357, 1991.

21. Henderson CE, Camu W, Mettling C, Gouin A, Poulsen K, Karihaloo M, et al. Neurotrophins promote motor neuron survival and are present in embryonic limb bud. Nature 363:266-270, 1993.

22. Lindsay RM. Brain-derived neurotrophic factor: an NGF related neurotrophin. In: Neurotrophic factors (Fallon JH, Loughlin SE, eds), pp 257-284. New York: Academic Press, 1993.

23. Mitsumoto H, Ikeda K, Klinkosz B, Cedarbaum JM, Wong V, Lindsay RM. Arrests of motor neuron disease in Wobbler mice cotreated with CNTF and BDNF. Science 265:1107-1110, 1994.

24. Yan Q, Elliott J, Snider WD. Brain-derived neurotrophic factor rescues spinal motor neurons from axotomy-induced cell death. Nature 360:753-755, 1992.

25. Tsukahara T, Yonekawa MD, Tanaka K, Ohara O, Watanabe S, et al. The role of brain-derived neurotrophic factor in transient forebrain ischemia in the rat brain. Neurosurgery 34:323, 1994.

26. Bradley WG. A phase I/II study of recombinant brain-derived neurotrophic factor in patients with ALS. Ann Neurol 38:971, 1995.
27. The BDNF Study Group (Phase III). A controlled trial of recombinant methionyl human BDNF in ALS. Neurology 52:1427-1433, 1999.

28. Ochs G, Penn RD, York M, Giess R, Beck M, Tonn J, et al. A phase I/II trial of recombinant methionyl human brain derived neurotrophic factor administrated by intrathecal infusion to patients with amyotrophic lateral sclerosis. Amyotroph Lateral Scler Other Motor Neuron Disord 1:201-206, 2000.

29. Pardridge WM, Kang Y-S, Buciak JL. Transport of human recombinant brain-derived neurotrophic factor (BDNF) through the rat blood-brain barrier in vivo using vector-mediated peptide drug delivery. Pharma Res 11:738-746, 1994.

30. Johnson DE, Williams LT. Structural and functional diversity in the FGF receptor multigene family. Adv Cancer Res 60:1-41, 1993.

31. Cuevas P, Carceller F, Ortega S, Zazo M, Nieto I, GimenezGallego G. Hypotensive activity of fibroblast growth factor. Science 254:1208-1210, 1991.

32. Rosenblatt S, Irikura K, Caday CG, Finklestein SP, Moskowitz MA. Basic fibroblast growth factor dilates rat pial arterioles. J Cereb Blood Flow Metab 14:70-74, 1994.

33. Ay H, Ay I, Joroshetz WJ, Finklestein SP. Potential usefulness of basic fibroblast growth factor as a treatment for stroke. Cerebrovascular Dis 9:131-135, 1999.

34. Deguchi Y, Naito T, Yuga T, Furukawa A, Yamada S, Pardridge WM, et al. Blood-brain barrier transport of I-125 labeled fibroblast growth factor. Pharma Res 17:63-69, 2000.

35. The FIBLAST Safety Study Group. Clinical safety of intravenous bFGF in acute stroke. Stroke 29:287, 1998.

36. Bogousslavsky J, Victor SJ, Salinas EO, Pallay A, Donnan GA, Fieschi C, et al. Fiblast (trafermin) in acute stroke: results of the European-Australian phase II/III safety and efficacy trial. Cerebrovas Dis 14:239-251, 2002.

37. Jeffries WA, Brandon MR, Hunt SV, Williams AF, Gatter KC, Mason DY. Transferrin receptor on endothelium of brain capillaries. Nature 312:162-163, 1984.

38. Pardridge WM. Blood-brain barrier drug targeting enables neuroprotection in brain ischemia following delayed intravenous administration of neurotrophins. Adv Exp Med Biol 513:397-430, 2002.

39. Wu D, Pardridge WM. Central nervous system pharmacologic effect in conscious rats after intravenous injection of a biotinylated vasoactive intestinal peptide analog coupled to a blood-brain barrier drug delivery system. J Pharmacol Exper Ther 279:77-83, 1996.

40. Wu D, Pardridge WM. Neuroprotection with noninvasive neurotrophin delivery to the brain. Proc Natl Acad Sci USA 96:254-259, 1999.

41. Wu D, Song B-W, Vinters HV, Pardridge WM. Pharmacokinetics and brain uptake of biotinylated basic fibroblast growth factor conjugated to a blood-brain barrier drug delivery system. J Drug Target 10:239-245, 2002.

42. Zhang Y, Pardridge WM. Conjugation of brain-derived neurotrophic factor (BDNF) to a blood-brain barrier targeting system enables neuroprotection in regional brain ischemia following intravenous injection of the neurotrophin. Brain Res 889:49-56, 2001.

43. Zhang Y, Pardridge WM. Neuroprotection in transient focal brain ischemia after delayed intravenous administration of brain-derived neurotrophic factor conjugated to a blood-brain barrier drug targeting system. Stroke 32:1378-1384, 2001.

44. Said SI, Mutt V. Polypeptide with broad biological activity. Isolation from small intestine. Science 169:1217-1218, 1970.

45. Suzuki Y, McMaster D, Lederis K, Rorstad OP. Characterization of relaxant effects of vasoactive intestinal peptide (VIP) and PHI on isolated brain arteries. Brain Res 322:9-16, 1984.

46. Yaksh TL, Wang J-Y, Go VLW. Cortical vasodilatation produced by vasoactive intestinal peptide (VIP) and by physiological stimuli in the rat. J Cereb Blood Flow Metab 7:315-326, 1987.

47. Brenneman DE, Gozes I. A femtomolar-acting neuroprotective peptide. J Clin Invest 97:2299-2307, 1996.

48. Brenneman DE, Westbrook GL, Fitzgerald SP, Ennist DL, Elkins KL, Ruff MR, et al. Neuronal cell killing by the envelope protein HIV and its prevention by vasoactive intestinal peptide. Nature 335:639-642, 1988. 
49. Gozes I, Lilling G, Glazer R, Ticher A, Ashkenazi IE, Davidson A, et al. Superactive lipophilic peptides discriminate multiple vasoactive intestinal peptide receptors. J Pharmacol Exper Ther 273: 161-167, 1995.

50. McCulloch J, Edvinsson L. Cerebral circulatory and metabolic effects of vasoactive intestinal polypeptide. Am J Physiol (Lond) 238:H449-H456, 1980.

51. Bickel U, Yoshikawa T, Landaw EM, Faull KF, Pardridge WM. Pharmacologic effects in vivo in brain by vector-mediated peptide delivery. Proc Natl Acad Sci USA 90:2618-2622, 1993.

52. Kang Y-S, Pardridge WM. Use of neutral-avidin improves pharmacokinetics and brain delivery of biotin bound to an avidinmonoclonal antibody conjugate. J Pharmacol Exper Ther 269: 344-350, 1994.

53. Kang Y-S, Saito Y, Pardridge WM. Pharmacokinetics of $[3 \mathrm{H}] \mathrm{bi}-$ otin bound to different avidin analogues. $J$ Drug Target 3:159$165,1995$.

54. Pardridge WM, Wu D, Sakane T. Combined use of carboxyldirected protein pegylation and vector-mediated blood-brain barrier drug delivery system optimizes brain uptake of brain-derived neurotrophic factor following intravenous administration. Pharma Res 15:576-582, 1998.

55. Schabitz W-R, Sommer C, Zoder W, Kiessling M, Schwaninger $\mathrm{M}$, Schwab S. Intravenous brain-derived neurotrophic factor reduces infarct size and counterregulates Bax and Bcl-2 expression after temporary focal cerebral ischemia. Stroke 31:2212-2217, 2000.

56. Song B-W, Vinters HV, Wu D, Pardridge WM. Enhanced neuroprotective effects of basic fibroblast growth factor in regional brain ischemia after conjugation to a blood-brain barrier delivery vector. J Pharmacol Exper Ther 301:605-610, 2002.

57. Berger M, Shankar V, Vafai A. Therapeutic applications of monoclonal antibodies. Am J Med Sci 324:14-30, 2002.

58. Shin SU, Wu D, Ramanathan R, Pardridge WM, Morrison SL.
Functional and pharmacokinetic properties of antibody-avidin fusion proteins. J Immunol 158:4797-4804, 1997.

59. Pardridge WM. Brain drug targeting. The future of brain drug development. Cambridge, UK: Cambridge University Press, 2001.

60. Wu D, Yang J, Pardridge WM. Drug targeting of a peptide radiopharmaceutical through the primary blood-brain barrier in vivo with a monoclonal antibody to the human insulin receptor. J Clin Invest 100:1804-1812, 1997.

61. Coloma MJ, Lee HJ, Kurihara A, Landaw EM, Boado RJ, Morrison SL, Pardridge WM. Transport across the primate blood-brain barrier of a genetically engineered chimeric monoclonal antibody to the human insulin receptor. Pharm Res 17:266-274, 2000.

62. Ataus SA, Onal MZ, Ozdem SS, Locke KW, Balkan S. The effects of citicoline and lamotrigine alone and in combination following permanent middle cerebral artery occlusion in rats. Int $J$ Neurosci 114:183-196, 2004.

63. Bradberry JC, Fagan SC, Gray DR, Moon YS. New perspectives on the pharmacotherapy of ischemic stroke. J Am Pharm Assoc 44[2 Suppl 1]:S46-S56, 2004.

64. Chen SD, Lee JM, Yang DI, Nassief A, Hsu CY. Combination therapy for ischemic stroke: potential of neuroprotectants plus thrombolytics. Am J Cardiovasc Drugs 2:303-313, 2002.

65. Sobrado M, Lopez MG, Carceller F, Garcia AG, Roda JM. Combined nimodipine and citicoline reduce infarct size, attenuation apoptosis and increase bcl-2 expression after focal cerebral ischemia. Neuroscience 118:107-113, 2003.

66. Nappi J, Talbert R. Dual antiplatelet therapy for prevention of recurrent ischemic events. Am J Health Syst Pharm 59:1723-1735, 2002.

67. Schabitz WR, Li F, Irie K, Sandage BW Jr, Locke KW, Fisher M. Synergistic effects of a combination of low-dose basic fibroblast growth factor and citicoline after temporary experimental focal ischemia. Stroke 30:427-431, 1999. 